\title{
Influence of the neutron transport tube on neutron resonance densitometry
}

\author{
Fumito Kitatani $^{1, \text { a }}$, Harufumi Tsuchiya ${ }^{1}$, Mitsuo Koizumi ${ }^{1}$, Jun Takamine ${ }^{1}$, Junichi Hori ${ }^{2}$, and Tadafumi Sano ${ }^{2}$ \\ 1 Japan Atomic Energy Agency, Tokai-mura, Ibaraki 319-1195, Japan \\ 2 Kyoto University Research Reactor Institute, Kumatori-cho, Osaka 590-0497, Japan
}

\begin{abstract}
Neutron Resonance Densitometry (NRD) is a non-destructive assay technique of nuclear materials in particle-like debris that contains various materials. An aim of NRD is to quantify nuclear materials in a melting fuel of Fukusima Daiichi plant, spent nuclear fuel and annihilation disposal fuel etc. NRD consists of two techniques of Neutron Resonance Transmission Analysis (NRTA) and Neutron Resonance Capture Analysis (NRCA) or Prompt Gamma-ray Analysis (PGA). A density of nuclear material isotopes is decided with NRTA. The materials absorbing a neutron in a wide energy range such as boron in a sample are identified by NRCA/PGA. The information of NRCA/PGA is used in NRTA analysis to quantify nuclear material isotopes. A neutron time of flight (TOF) method is used in NRD measurements. A facility, consisting of a neutron source, a neutron flight path, and a detector is required. A short flight path and a strong neutron source are needed to downsize such a facility and put NRD into practical use. A neutron transport tube covers a flight path to prevent noises. In order to investigate the effect of neutron transport tube and pulse width of a neutron source, we carried out NRTA experiments with a 2-m short neutron transport tube constructed at Kyoto University Research Reactor Institute - Linear Accelerator (KURRI-LINAC), and impacts of shield of neutron transport tube and influence of pulse width of a neutron source were examined. A shield of the neutron transport tube reduced a background and had a good influence on the measurement. The resonance dips of ${ }^{183} \mathrm{~W}$ at $27 \mathrm{eV}$ was successfully observed with a pulse width of a neutron source less than $2 \mu \mathrm{s}$.
\end{abstract}

\section{Introduction}

Neutron Resonance Densitometry (NRD) [1,2] is an assay technique of nuclear materials in particle-like debris that contains various materials. As for the object sample, there is a melting fuel of Fukusima Daiichi plant, spent nuclear fuel and annihilation disposal fuel etc. It is a combination of two techniques: Neutron Resonance Transmission Analysis (NRTA) [3], and Neutron Resonance Capture Analysis (NRCA) [4] or Prompt Gamma-ray Analysis (PGA). A density of nuclear material isotopes is decided with NRTA. The materials absorbing a neutron in a wide energy range such as boron a in a sample are identified by NRCA/PGA. The information of NRCA/PGA is used in NRTA analysis to quantify nuclear material isotopes.

A neutron time of flight (TOF) method is used in NRD measurements. A facility consisting of a neutron source, a neutron flight path, and a detector, is required. A highly precise measurement and a small device are necessary to perform practical use of NRD. Therefore, a strong neutron source and a system of a low noise are necessary for a device. In order to investigate the effect of neutron transport tube and pulse width of a neutron source, we carried out NRTA experiments at Kyoto University Research Reactor Institute - Linear Accelerator (KURRILINAC).

We have examined NRTA with a 7.5-m flight path having 2-m neutron transport tube. It was checked about

\footnotetext{
a e-mail: kitatani.fumito@jaea.go.jp
}

influence of shield of a neutron transport tube and influence of a pulse width of a neutron source.

\section{Experiment}

Experiments were carried out with a 7.5-m flight path and 2-m neutron transport tube at KURRI-LINAC. The experimental setup is shown in Fig. 1. An experimental room and a neutron source are separated by a 2-m-thick concrete wall. An electron beam bombards a Ta target, producing neutrons via $(\gamma, \mathrm{n})$. The produced neutrons are moderated by $\mathrm{H}_{2} \mathrm{O}$. An aperture with a diameter of $5 \mathrm{~cm}$ and a length of $30 \mathrm{~cm}$, made of borated polyethylene, is installed in a source side of the concrete wall, collimating neutron beams to $50 \mathrm{~mm}$ in diameter in front of a neutron transport tube with 2-m lengh. The neutron transport tube is installed in the experimental room side. Its center was an $\mathrm{Al}$ vacuum chamber, the cavity of which is $200 \mathrm{~mm}$ in diameter; both sides are shielded by 2 -mm-thick $\mathrm{Al}$ plates. The cylindrical surface of chamber is surrounded by 5-mm thick $\mathrm{B}_{4} \mathrm{C}$-doped silicone rubber $\left(\mathrm{B}_{4} \mathrm{C} 40 \mathrm{wt} \%\right)$. The outside of the rubber was high density polyethylene plates $(460 \times 460 \mathrm{~mm})$.

A ${ }^{6} \mathrm{Li}$ scintillation detector Saint-Gobain GS20 $\left(95 \%{ }^{6} \mathrm{Li}\right),(100 \times 100 \times 10 \mathrm{~mm})$ was used to measure transmission neutrons. The detector was covered with lead $(3 \mathrm{~cm})$ and borated polyethylene $(10 \mathrm{~cm})$. The incident neutron flux was monitored by a $\mathrm{BF}_{3}$ neutron detector placed near the exit of the concrete wall. 

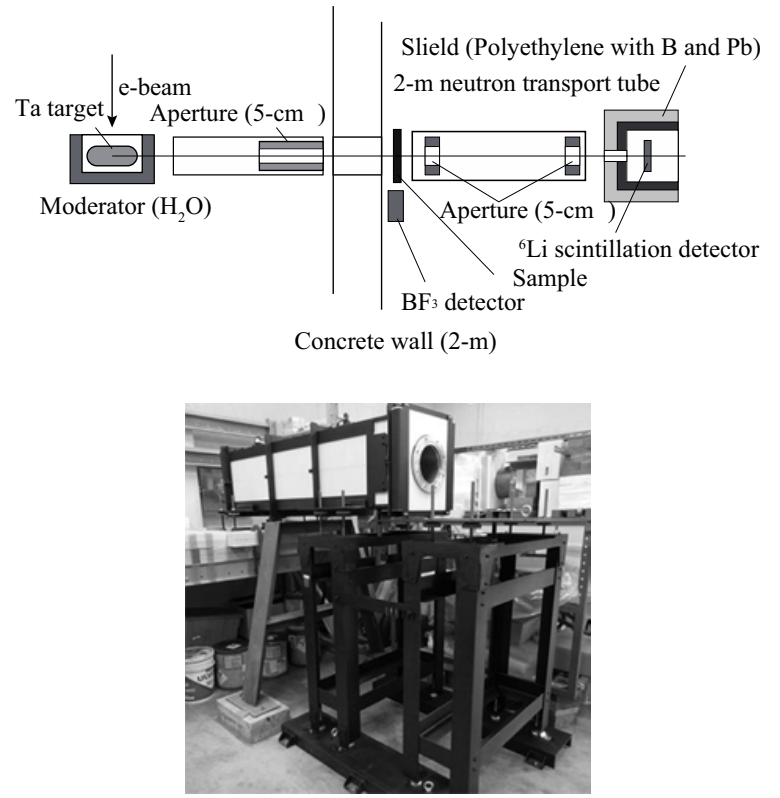

Figure 1. The set up of neutron source and the 2-m-long neutron transport tube.

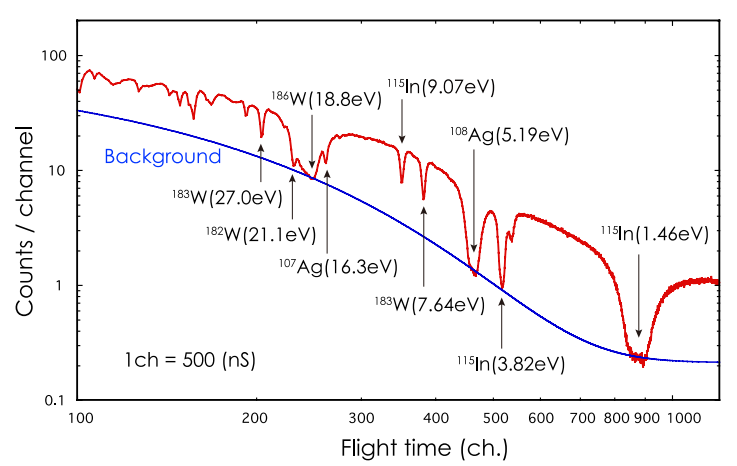

Figure 2. TOF neutron transmission spectrum with the 2-m neutron transport tube.

A sample plate $(100 \times 100 \mathrm{~mm})$ was placed in front of the transport tube: In $(0.2 \mathrm{~mm}), \mathrm{Ag}(0.25 \mathrm{~mm}), \mathrm{W}$ $(0.5 \mathrm{~mm})$, and $\mathrm{Pb}(10 \mathrm{~mm})$. The $\mathrm{Pb}$ was inserted for a gamma shield. The neutron pulse width was adjusted by changing the pulse width of the LINAC electron beams to $500 \mathrm{~ns}, 2 \mu \mathrm{s}$ or $4 \mu \mathrm{s}$.

\section{Results}

Figure 2 shows TOF neutron transmission spectrum with the 2-m neutron transport tube and with Ag-In-W plates. This spectrum contains background that is mainly due to scattering neutrons. Thus, the background should be removed from this spectrum in the following way. Using saturated absorption dips such as $1.46 \mathrm{eV}, 3.82 \mathrm{eV}, 5.19 \mathrm{eV}$ and $18.8 \mathrm{eV}$ the background was decided by fitting the next expression to those.

$$
B(t)=b_{0}+b_{1} e^{-\lambda t}
$$

Here, the first term represents the time independent contribution, and the second term corresponds to the time dependence of the background.

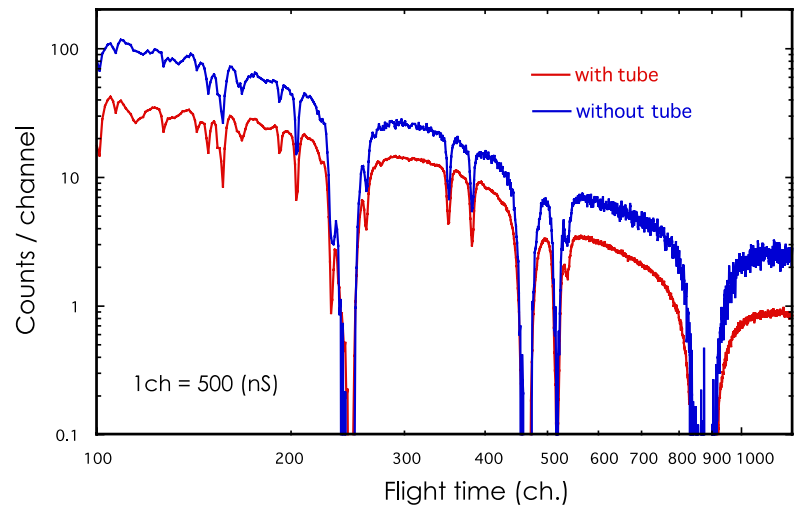

Figure 3. TOF neutron transmission spectra with and without the neutron transport tube.
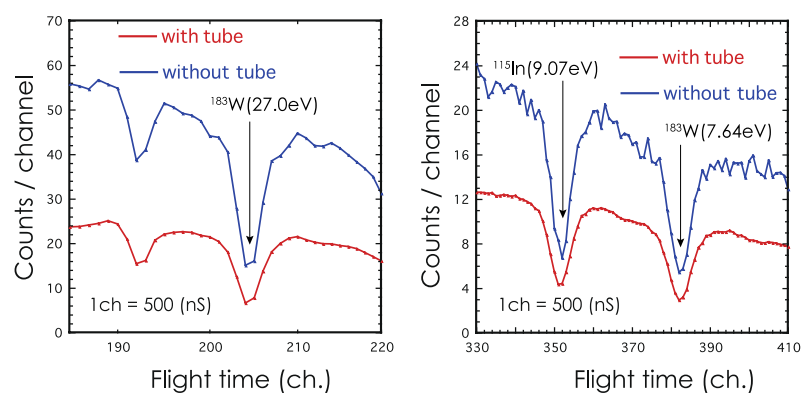

Figure 4. The absorption dips of ${ }^{183} \mathrm{~W}$ and ${ }^{115} \mathrm{In}$

Figure 3 compares TOF spectra with and without the neutron transport tube. The spectra were normalized by using the counts of $\mathrm{BF}_{3}$ detector set at an exit of 5-m neutron transport tube.

The backgrounds are removed from these spectra in the same way as Fig. 3. Even if the background is removed from the spectrum without the neutron transport tube, additional background contribution due to presence of the tube exist. Because there is no shield with the neutron transport tube, the many noises of the background cannot be removed enough.

Figure 4 shows several resonance dips observed with and without the tube.

From the figure, it is found that there are large differences in the depth of dip and the energy dependence of neutron intensity between the cases with and without the neutron transport tube.

Then, influence of the neutron pulse width on measured resonance dips was examined with the pulse width of electron beam changed to $0.5 \mu \mathrm{s}, 2 \mu \mathrm{s}$ or $4 \mu \mathrm{s}$. Generally, a TOF measurement decides the neutron energy by the time of flight. The relation of the flight time and the energy of neutron is expressed in Eq. (2).

$$
t=72.3 \cdot L / \sqrt{E}
$$

$t$ : flight time ( $\mu \mathrm{s}), L$ : distance $(\mathrm{m})$,

$E$ : energy of neutron $(\mathrm{eV})$.

If the pulse width of the neutron becomes longer, the energy resolution of the neutron becomes worse, and 

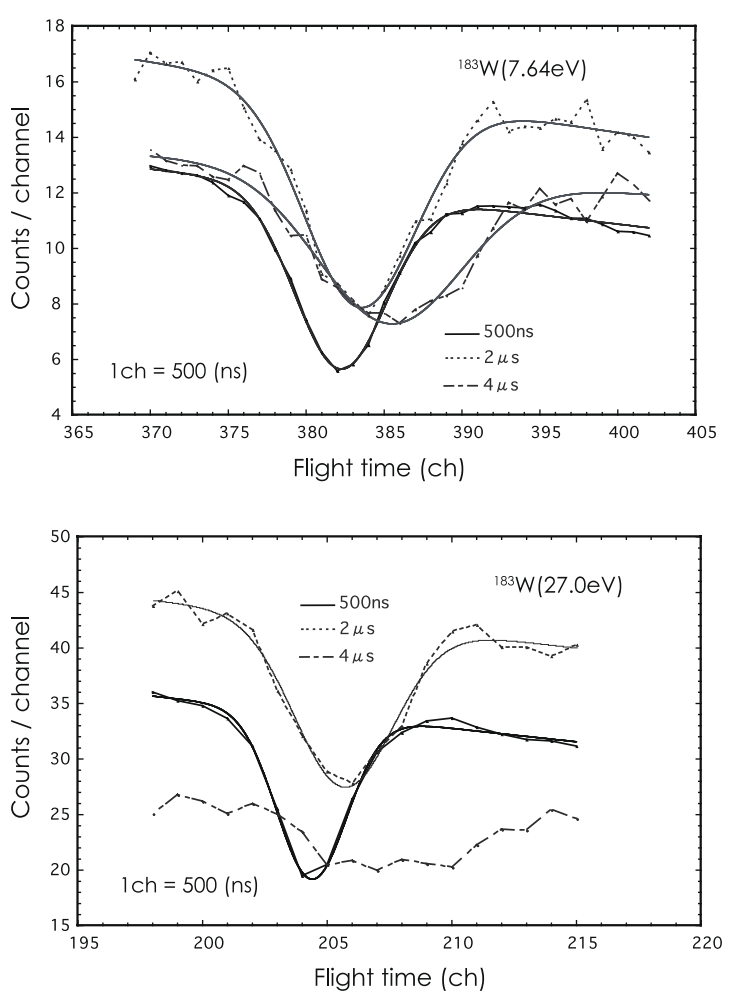

Figure 5. The two absorption dips of ${ }^{183} \mathrm{~W}$. The experimental and fitting curves are shown.

Table 1. Dip ch. and FWHM of the absorption dips.

\begin{tabular}{|c|c|c|}
\hline \multicolumn{3}{|c|}{$7.64 \mathrm{eV}$} \\
\hline $\begin{array}{c}\text { The pulse width of } \\
\text { electron beam }\end{array}$ & Dip channel & FWHM \\
\hline $0.5 \mu \mathrm{s}$ & 382.2 & 4.1 \\
\hline $2 \mu \mathrm{s}$ & 383.4 & 5.1 \\
\hline $4 \mu \mathrm{s}$ & 385.4 & 6.5 \\
\hline \multicolumn{3}{|c|}{$27.0 \mathrm{eV}$} \\
\hline $\begin{array}{c}\text { The pulse width of } \\
\text { electron beam }\end{array}$ & Dip channel & FWHM \\
\hline $0.5 \mu \mathrm{s}$ & 204.4 & 2.0 \\
\hline $2 \mu \mathrm{s}$ & 205.6 & 2.8 \\
\hline $4 \mu \mathrm{s}$ & - & - \\
\hline
\end{tabular}

consequently the measurement accuracy also becomes worsen. The absorption dips of ${ }^{183} \mathrm{~W}(7.64 \mathrm{eV})$ and ${ }^{183} \mathrm{~W}(27.0 \mathrm{eV})$ are shown in Fig. 5.
The resultant dip channel and FWHM of individual absorption dips are shown in Table 1. As shown in Table 1, the width of the absorption dip becomes broader as the neutron pulse width becomes longer. It was found that the absorption dip at $7.64 \mathrm{eV}$ clearly appeared under any neutron pulse widths, while that at $27.0 \mathrm{eV}$ did not appear under $4 \mu \mathrm{s}$ width. For a check, we computed dip channel and FWHM of each resonance dip by fitting.

\section{Summary}

In measurements of $\mathrm{NRD}$, the quantity of the nuclear materials is determined with a NRTA spectrum in the neutron energy range less than $30 \mathrm{eV}[1,2]$.

At KURRI-LINAC, we have performed NRTA with a 7.5-m flight path having 2-m neutron transport tube. The background subtraction is necessary for improvement of the measurement accuracy. Because the neutron transport tube shields backgrounds, it is indispensable for improving measurement accuracy.

The influence of the pulse width of the neutron was checked. The resonance dips of ${ }^{183} \mathrm{~W}$ at $27.0 \mathrm{eV}$ was successfully observed with an electron pulse width less than $2 \mu \mathrm{s}$.

This indicates the applicability of short-flight-path TOF system to nuclear material quantification. The present work showed that the importance of the pulse width of neutron beam. Further simulation studies and experiments are expected for a realistic design of a TOF facility of nuclear material quantification.

\section{References}

[1] H. Harada et al., Nuclear Data Sheets 118, 502 (2014)

[2] F.Kitatani et al., J. Nucl Sci. Tech. 51, 1107 (2014)

[3] P. Schillebeeckx et al., Nuclear Data Sheets 113, 3054 (2012)

[4] P. Schillebeeckx et al., JINST 7, C03009 (2012)

[5] P. Schillebeeckx et al., JRC science and policy reports Neutron resonance spectroscopy for the characterisation of materials and objects (EUR 26848 EN) (2014) 